\title{
Exercise and Environmental Enrichment as Enablers of Task-Specific Neuroplasticity and Stroke Recovery
}

\author{
Jessica Livingston-Thomas ${ }^{1,2}$ • Paul Nelson ${ }^{1,2}$ - Sudhir Karthikeyan ${ }^{1,2}$. \\ Sabina Antonescu ${ }^{1,2}$ - Matthew Strider Jeffers ${ }^{1,2}$. Susan Marzolini ${ }^{1,3}$. \\ Dale Corbett ${ }^{1,2,4,5}$
}

Published online: 11 February 2016

(C) The American Society for Experimental NeuroTherapeutics, Inc. 2016

\begin{abstract}
Improved stroke care has resulted in greater survival, but $>50 \%$ of patients have chronic disabilities and $33 \%$ are institutionalized. While stroke rehabilitation is helpful, recovery is limited and the most significant gains occur in the first 23 months. Stroke triggers an early wave of gene and protein changes, many of which are potentially beneficial for recovery. It is likely that these molecular changes are what subserve spontaneous recovery. Two interventions, aerobic exercise and environmental enrichment, have pleiotropic actions that influence many of the same molecular changes associated with stroke injury and subsequent spontaneous recovery. Enrichment paradigms have been used for decades in adult and neonatal animal models of brain injury and are now being adapted for use in the clinic. Aerobic exercise enhances motor recovery and helps reduce depression after stroke. While exercise attenuates many of the signs associated with normal aging (e.g., hippocampal atrophy), its ability to reverse cognitive impairments subsequent to stroke is less evident. It may be that stroke, like other diseases such as cancer, needs to use multimodal treatments that augment complimentary neurorestorative processes.
\end{abstract}

Dale Corbett

dcorbett@uottawa.ca

1 Canadian Partnership for Stroke Recovery, University of Ottawa, Ottawa, ON, Canada

2 Department of Cellular and Molecular Medicine, Faculty of Medicine, University of Ottawa, Ottawa, ON, Canada

3 Toronto Rehabilitation Institute, University Health Network, Toronto, ON, Canada

4 Faculty of Medicine, Memorial University of Newfoundland, St. John's, NL, Canada

5 Faculty of Medicine, University of Toronto, Toronto, ON, Canada
Key Words Neuroplasticity · exercise · enrichment · stroke · recovery of function

\section{Introduction}

Behavioral experience can markedly alter structural properties of neurons (e.g., alter spine formation, dendritic and axonal sprouting), as well as molecular processes such as neurogenesis and angiogenesis in the normal rodent brain [1-3]. In the first days and weeks following experimental stroke there is a period of heightened neuroplasticity characterized by an upregulation of growth-promoting genes and proteins [4]. During this time interval, behavioral interventions such as rehabilitation might be expected to be highly effective, since the molecular processes of self-repair that manifest as spontaneous recovery likely overlap with the neurorestorative mechanisms induced by rehabilitation. Evidence from rodent models of stroke support the concept of a time-limited window or "critical period" that is coincident with this neuroplastic time window, as rehabilitation delivered within the first few weeks after stroke, but not at later times, increases motor recovery and results in profound changes in dendritic complexity of cortical motor neurons [5]. Preclinical studies have employed different approaches to enhance recovery from brain injury, including stroke. One effective intervention is environmental enrichment (EE), a form of rehabilitation in which groups of animals are housed in complex environments consisting of a large cage containing ladders, beams, ramps, and a variety of toys to encourage sensorimotor stimulation $[1,2,6,7]$. By introducing novel objects and/or by periodically rearranging the objects within the EE cage a degree of cognitive stimulation can be achieved (Fig. 1a). Collectively, EE provides an environment that promotes physical and cognitive activity, as well as social interaction. Most 
Fig. 1 (a) A typical enriched environment cage containing a variety of colored and textured toys, platforms, and other objects to encourage exploration and sensorimotor stimulation. By introducing novel objects or locating them in different places an element of cognitive stimulation can be achieved in addition to social interaction. (b) Poststroke exercise is most often administered using voluntary or motorized running wheels or treadmills. (c) Exercise and enriched environment induce multiple neuroplastic processes. Resulting changes, such as the reorganization of surviving cortical and subcortical circuits, ultimately promote poststroke functional recovery. GAP-43 = growth-associated protein 43; MAP2 $=$ microtubule-associated protein 2; MMP2 = matrix metalloproteinase 2; VEGF = vascular endothelial growth factor; $\mathrm{BDNF}=$ brain-derived neurotrophic factor
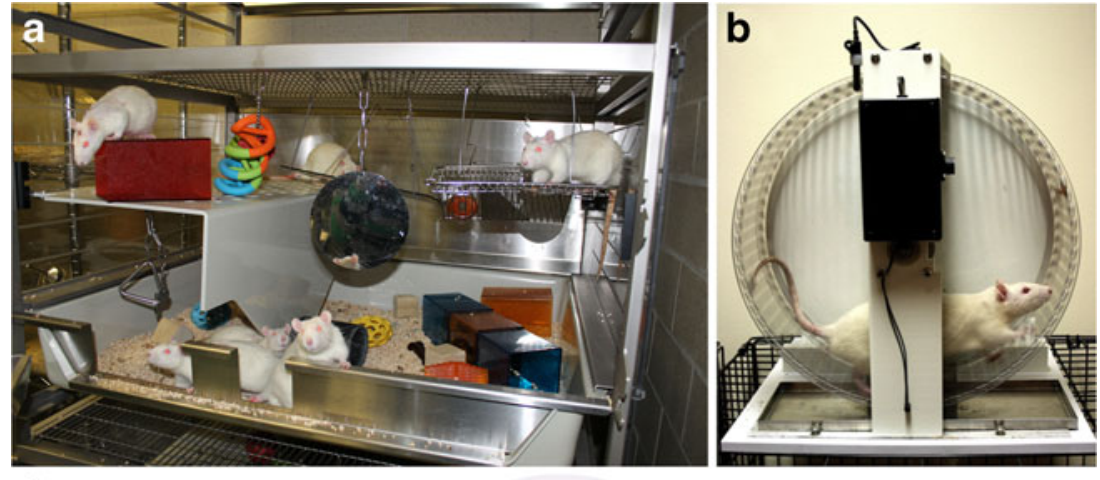

C

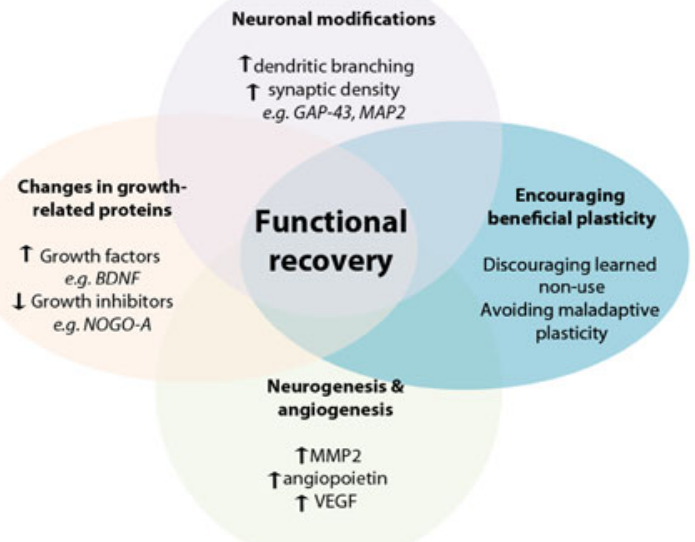

stroke patients in the days and weeks following stroke are alone approximately $60 \%$ of the time and during waking hours are physically inactive nearly $75 \%$ of the time [8]. Indeed, the contrast between EE and the human poststroke environment with respect to the amount of stimulation, physical exercise, and socialization is striking in animal studies providing earlier and more intense rehabilitation [9]. Fortunately, clinical rehabilitation protocols are being developed that incorporate exercise and aspects of EE, but the process has been slow and is by no means standard practice in most stroke rehabilitation centers.

Here we review preclinical and clinical evidence showing how $\mathrm{EE}$ and exercise enable neuroplasticity processes to achieve poststroke motor and cognitive recovery. In addition, we discuss how the efficacy of such interventions changes over the lifespan of the organism, and conclude by providing some suggestions for future translational research.

\section{EE, Exercise, and Stroke Recovery}

The ability of EE and other forms of behavioral experience to induce neuroplastic changes with resulting behavioral modification is well documented [1, 3, 10]. However, Johansson [6] was the first to exploit EE to treat the stroke-damaged brain. In a series of papers, Johansson and colleagues showed that EE improved recovery of general neurological function (e.g., limb placing, balance) when initiated in the first days and weeks after focal stroke in rats [6]. As EE is comprised of several different components (socialization, exercise, sensorimotor and cognitive stimulation) a question that often arises is which element(s) is responsible for the improvement in postinjury recovery? Experiments that dissect the individual elements of EE (e.g., exercise vs socialization) show that EE is superior to any one of the components, including exercise, in promoting recovery from brain injury $[2,11]$. Another important issue concerns the generalizability or specificity of the actions of EE on behavior. At one level of analysis it seems that EE is universally effective; it enhances sensorimotor behaviors, learning and memory, and other cognitive and psychosocial functions across many different types and sites of brain injury [2]. However, while EE reduces impairments in some tasks following frontal cortex lesions, it does not reverse deficits in certain species-typical behaviors such as food hoarding and grooming [12]. Further, analysis of many stroke studies indicates that $\mathrm{EE}$ is effective in reducing general neurological impairments (e.g., limb placing, balance) but has limited efficacy in attenuating deficits in skilled reaching following stroke [13]. Seemingly, EE by itself does not provide the task-specific training necessary for relearning skilled forelimb movements such as grasping. For this reason, we developed a rehabilitation paradigm combining EE with daily reach training sessions [14]. This paradigm, which we termed enriched rehabilitation (ER) to distinguish it from standard 
$\mathrm{EE}$, is very effective in enhancing recovery of upper limb function (and other functions) following damage to forelimb motor cortex regions and as such represents what might be considered preclinical "best practice" [7, 15]. Training of the upper limb results in use-dependent growth of cortical dendritic arbor that correlates with recovery of forelimb function $[13,16]$. Similarly, rats housed in EE show increased formation of dendritic branches and spines in layer II/III motor neurons compared with standardly housed animals [17]. However, it is important to note that ER produces a more selective and more robust use-dependent activation of layer II/III motor neurons in peri-infarct cortex, as revealed by activation of the immediate early gene, $F o s B / \Delta F o s B$ [18], than $\mathrm{EE}$ or reach training (individual components of ER). In fact, the latter interventions were no more effective than standard housing in inducing $F o s B / \triangle F o s B$ expression. As cortical layer II/III neurons have extensive cortico-cortical interconnections, neuroplastic changes in this region produced by ER may be important for the neural remodeling contributing to stroke recovery [19].

An important distinction between preclinical and clinical stroke exercise studies is that animal studies are invariably initiated early ( $1-2 \mathrm{~h}$ to several days, poststroke) in the acute or subacute phase after stroke and have focused mainly on neuropathological outcomes (e.g., changes in infarct volume) [20]. In contrast, exercise interventions in patients are often conducted in the chronic phase of stroke (i.e., $>6$ months) or when done early as part of inpatient services, exercise overlaps with usual care (i.e., rehabilitation) and hence is an adjunctive therapy. In animal studies exercise can be either voluntary (e.g., wheel running) or forced, using motorized treadmills or running wheels (Fig. 1b). Unlike human studies, animal studies rarely set a training intensity goal such as a percentage of maximal heart rate or maximal oxygen uptake $\left(\mathrm{VO}_{2}\right.$ max). Herein, we did not include preclinical exercise studies initiated earlier than $24 \mathrm{~h}$ poststroke, because cell death is not complete at this time [21].

A perusal of the animal literature reveals that very few investigators have initiated exercise beyond the time when the stroke injury may still be evolving (i.e., 1-3 days poststroke); consequently, such studies are confounded as it is impossible to determine if improvement in behavioral outcome is due to a neuroprotective or a neurorestorative effect, or both. In one study young and middle-aged female rats had access to $6 \mathrm{~h}$ of wheel running per day alone or in combination with daily reach training. Only reach training facilitated recovery of skilled reaching following sensorimotor cortex lesions. Running exercise did not improve recovery, nor did it add to the benefit provided by reach training [22]. In another study, 18-month-old rats were subjected to daily walking in motorized running wheels beginning 7 days after an endothelin-1induced stroke of sensory cortex. The exercise protocol mirrored clinical studies in that it became progressively more challenging over the 4-week exercise period. Results show that exercise facilitated somatosensory recovery in the absence of any effect on neurogenesis [23]. Like EE, physical exercise improves recovery of general neurological function (e.g., limb placing, balance) [24, 25] but not motor movements requiring a high level of dexterity such as reaching in rodents after stroke.

The human literature provides more compelling evidence for the ability of aerobic exercise to improve motor recovery after stroke [26], and this information has culminated in an American Heart Association position paper outlining exercise recommendations for stroke survivors [27]. A particularly successful exercise program is the Fitness and Mobility Exercise protocol that has achieved significant improvement in muscle strength, balance, walking speed, cardiovascular fitness, and bone density [28]; however, questions concerning the optimal dose, frequency, duration, and therapeutic time window of exercise remain. Initial studies suggest that combining aerobic exercise with resistance training appears to be an effective means for improving poststroke cognition, including executive function [29]. Combination therapies may prove useful in treating other stroke-related functional impairments.

Stroke recovery research has primarily focused on restoring lost motor function. However, deficits in cognition and psychosocial function, including depression, affect $40-60 \%$ of individuals living with stroke [30-32]. This area of research has received little attention, especially in the preclinical setting. Therefore, a concentrated effort to understand the mechanisms underlying recovery of cognitive function and normalization of mood is urgently needed in order to develop new treatments.

As with motor function, housing rodents in EE improves cognitive functions in global and focal stroke models [33-35]. Improvement in cognitive function is correlated with changes in plasticity-related genes and growth factors [33], neurogenesis [36], and endothelial cell proliferation [37]. Aerobic exercise has also been shown to induce cortical angiogenesis and improve executive function in middle-aged Cynomolgus monkeys [38].

As exercise and enrichment alter processes that are modulated by antidepressant drugs [e.g., elevation of brain-derived neurotrophic factor (BDNF)], it is not surprising that exercise and enrichment attenuate depression and anxiety. Clinically, poststroke exercise appears to prevent or reduce depressive symptoms in both subacute and chronic patients [39]. Promisingly, enrichment reverses depression-like behavior and increases cell proliferation and survival in an uninjured depression model [40], mechanisms that may translate to poststroke improvements.

In the studies highlighted herein, animals were housed in EE beginning within the first week poststroke, and subsequently exhibited improved spatial memory and increased markers of neuroplasticity in the ensuing weeks [33-37]. 
Rehabilitation of motor function is subject to a permissive neuroplastic time window following injury, during which there are alterations in the regulation of both growth promoting and growth inhibiting factors [4]. The existence of a similar "growth-permissive" period may exist with respect to cognition. Clinically, spontaneous recovery of cognitive function continues beyond the 3-4-month poststroke period when motor recovery plateaus [41], suggestive of a broader cognitive therapeutic time window. The development of stroke models that target cognitive function will hopefully lead to new insights into the nature of poststroke cognitive and psychosocial recovery $[42,43]$.

\section{How do Exercise and Enrichment Enhance Plasticity and Recovery?}

The growth-permissive poststroke epoch, which lasts for approximately 30 days in rodents, is associated with the greatest potential for functional recovery and is later followed by a temporal increase in the regulation of growth-inhibitory factors that limit recovery [4]. Poststroke exercise and EE have the potential to augment endogenous brain plasticity processes and enhance functional recovery, either alone or in conjunction with other therapies (Fig. 1C).

The most well-characterized growth factor linked to poststroke recovery is BDNF. Following stroke, aerobic exercise increases serum BDNF levels in patients [44], and effectively enhances recovery [29, 44]. Similarly, preclinical models have demonstrated that poststroke exercise and EE both increase cortical and hippocampal BDNF expression and improve recovery [33, 45-47]. Most importantly, blocking BDNF attenuates spontaneous recovery and the effects of poststroke rehabilitation in rodent models of stroke $[47,48]$.

Poststroke exercise and EE also increase expression of growth-associated proteins such as growth-associated protein 43 [49], microtubule-associated protein 2 [44], and netrin (along with the associated deleted in colorectal cancer receptor) [50], as well as markers of synaptogenesis [46, 51, 52]. Such proteins are involved in axonal sprouting, dendritic complexity, and spine growth [4], phenomena occurring in the peri- and contralesional cortex of functionally recovered animals $[14,53]$.

Stroke induces neurogenesis and triggers migration of newly born cells to the site of damage [54]. Poststroke exercise and EE further augment these processes in various stroke models [36, 55-57]. Interestingly, Komitova et al. [57] reported that while poststroke EE enhanced neurogenesis in the subventricular zone, unlimited access to exercise beginning $24 \mathrm{~h}$ poststroke attenuated this effect. Notably, the effects of exercise and EE are difficult to separate, because most enrichment paradigms incorporate an element of exercise. In an attempt to separate the effects of each modality, Bechara and Kelly [58] and Grégoire et al. [59] used intact animals and found that exercise, but not EE, increased neurogenesis. This differential effect could be a result of differences in exercise intensity, and stress inherent to wheel-running and EE paradigms. Furthermore, the milieu of the poststroke brain may respond differently to activity-dependent interventions and associated stress, especially when initiated $24 \mathrm{~h}$ poststroke.

An integral component that contributes to the growthpermissive environment following stroke is a reduction of growth inhibitors. Growth inhibition is largely mediated by components of the extracellular matrix termed perineuronal nets (PNNs), and growth inhibitory proteins such as $\mathrm{Nogo}_{\mathrm{A}}$. Exposing animals to poststroke EE and exercise decreases the expression of PNNs and $\mathrm{Nogo}_{\mathrm{A}}$ in perilesional tissue [60, 61], potentially contributing to the observed functional recovery. Similarly, treating poststroke animals with anti- $\mathrm{Nogo}_{\mathrm{A}}$ antibody or PNN-digesting chondroitinase $\mathrm{ABC}$ is associated with enhanced axonal plasticity and enhanced recovery [62, 63]. Together, these findings suggest that exercise and EE contribute to the induction of a permissive environment for structural remodeling of neural networks by decreasing the involvement of growth inhibitory molecules.

Following stroke, reperfusion of perilesional tissue is critical for recovery. In intact animals, exercise and EE increase angiogenesis in the hippocampus, striatum, and cortex [3, 37]. Similarly, in the poststroke brain, exercise enhances vascular growth, contributing to sustained increases in microvasculature, cerebral blood flow, and functional recovery $[64,65]$.

While activity-dependent neuroplasticity can be beneficial for functional recovery it can also be detrimental. For example, following stroke humans spontaneously develop a reliance on their nonparetic limb $[65,66]$, which leads to learned nonuse of the affected limb and persistent compensatory behavior with further reduction in use of the impaired limb [67]. In rats, forced training of the nonimpaired forelimb has a negative effect on the recovery of the impaired $\operatorname{limb}[68,69]$. The mechanisms mediating this maladaptive plasticity appear to be associated with alterations in transcallosal connections from the contralesional cortex [70], decreased neuronal activation of the perilesional cortex [68], and aberrant synaptogenesis and reorganization of the perilesional tissue [71]. The detrimental effects of nonimpaired limb training can be overcome by using bilateral training [66], emphasizing that compensatory use of the unimpaired limb can drive maladaptive forms of plasticity and ultimately limit functional recovery.

\section{Therapeutic Interventions Across the Lifespan}

While stroke is more likely to occur with increasing age, it can occur at any age, leaving survivors with persistent motor and 
cognitive disabilities [72]. While some studies show that the immature brain is more vulnerable to brain damage resulting from stroke [73], others have suggested that increasing age has no effect on stroke volume [74], or may worsen infarct size [75]. Nevertheless, age affects many of the molecular processes commonly associated with neuroplasticity and stroke recovery.

Comparing efficacy of poststroke therapies between young and aged animals is difficult, owing to the paucity of preclinical research using senescent animals. In neonates, EE represents a promising therapeutic treatment. The immature brain is highly receptive to environmental stimuli; indeed, even gentle handling of rat pups for 15 min per day following neonatal injury improves long-term spatial learning ability [76]. Notably, in a neonatal model of hypoxia-ischemia, EE improves object recognition and spatial memory [77, 78], attributed in part to preservation of hippocampal dendritic spine density [77]. In aged rats, EE improves recovery of cognitive function following focal cerebral ischemia [79]. Furthermore, aging studies suggest that animals may require more intense stimulation or longer time in EE than younger animals in order to display similar beneficial effects [80]. Aged rats subjected to clinically relevant mild walking exercise also show enhanced somatosensory function following stroke, through unidentified, non-neurogenic mechanisms [23]. Elements of EE can be adapted for use in the clinic by providing a more stimulating environment, enriched by physical (adapted exercise equipment), social (communal activities such as cards, bingo, etc.), and cognitive (e.g. reading material, internet) activities. For example, tablet technology allows for patients to engage in cognitive exercises and games that can be tailored to their specific impairments and made progressively more challenging as they improve. Along these lines, Janssen et al. [81] have reported encouraging preliminary results in a study where EE was incorporated into a stroke rehabilitation unit.

Aged animals exhibit a slower and less complete poststroke recovery than their younger counterparts on both cognitive and motor tasks [73, 79, 82]. Interestingly, although aged rats are not able to recover as fully as younger rats on a single pellet reaching task, all ages use similar compensatory movements to improve their performance [83]. Clinically, advancing age appears to be related to declines in ability to recover from stroke if considered from the perspective of performing activities of daily living (ADL) [74]. However, by examining both ADL and the more neurologically focused Scandinavian Stroke Scale, Nakayama et al. [74] dissociated ADL performance (easily influenced by compensatory strategies) from true neurological improvement, which was not affected by age. Thus, age does not necessarily influence the ability to recover from the stroke per se, but rather may reflect differences in the compensatory motor strategies enacted by elderly stroke survivors. As such, recovery may be affected by factors such as decreases in overall mobility, which can affect the ability to successfully enact new movement strategies [74]. The differences in physical abilities of each person emphasize the importance of individually tailoring poststroke treatments.

In accordance with the STAIR recommendations, further research comparing poststroke treatment efficacy, as well as the neurological response, should be performed across a variety of subject ages [84]. This would allow the investigation of interactions between decreasing neuroplasticity and other factors that decline with age, such as mobility, and how they might be controlled to optimize recovery from stroke at different ages throughout the lifespan. In addition, the vast majority of exercise and enrichment studies have been conducted in healthy, male animals. Given the high incidence and adverse effects of disease comorbidity factors on stroke occurrence and outcomes it is imperative that future preclinical studies incorporate such factors into animal models to help mitigate translational failure to the clinic $[85,86]$. Similarly, there is much debate in the human stroke rehabilitation field about the influence of timing, duration, and intensity of rehabilitation $[9,27,87]$. These variables have received scant attention in preclinical exercise and enrichment studies, and thus would be a fruitful avenue for future investigation $[23,46,80]$.

\section{Summary}

Pleiotropic interventions such as exercise and EE to enhance motor recovery following stroke appear to hold great promise given the widespread failure of monotherapeutic approaches [88]. The same strategy may be beneficial for cognitive recovery but the research in this area is less advanced. As discussed, factors such as age, disease comorbidity, sex, and dose of exercise and enrichment can markedly influence functional outcome and need to be thoughtfully incorporated into animal models of stroke in order to minimize translational failure.

Acknowledgments This work was supported by operating grants to D.C. from the Canadian Institutes for Health Research (CIHR), the Heart and Stroke Foundation of Canada, and the Canadian Partnership for Stroke Recovery. D.C. was the recipient of a Tier 1 Canada Research Chair in Stroke and Neuroplasticity.

Required Author Forms Disclosure forms provided by the authors are available with the online version of this article.

\section{References}

1. Kolb B, Forgie M, Gibb R, Gorny G, Rowntree S. Age, experience and the changing brain. Neurosci Biobehav Rev 1998; 22:143-159.

2. Will B, Galani R, Kelche C, Rosenzweig MR. Recovery from brain injury in animals: relative efficacy of environmental enrichment, physical exercise or formal training (1990-2002). Prog Neurobiol 2004; 72:167-182. 
3. Kleim JA, Jones TA. Principles of experience-dependent neural plasticity: implications for rehabilitation after brain damage. $J$ Speech Lang Hear Res 2008; 51:S225-S239.

4. Carmichael ST, Archibeque I, Luke L, Nolan T, Momiy J, Li S. Growth-associated gene expression after stroke: evidence for a growth-promoting region in peri-infarct cortex. Exp Neurol 2005; 193:291-311.

5. Biernaskie J, Chernenko G, Corbett D. Efficacy of rehabilitative experience declines with time after focal ischemic brain injury. $\mathrm{J}$ Neurosci 2004; 24:1245-1254.

6. Johansson BB. Functional and cellular effects of environmental enrichment after experimental brain infarcts. Restor Neurol Neurosci 2004; 22:163-174.

7. Murphy TH, Corbett D. Plasticity during stroke recovery: from synapse to behaviour. Nat Rev Neurosci 2009; 10:861-872.

8. Bernhardt J, Dewey H, Thrift A, Donnan G. Inactive and alone: physical activity within the first 14 days of acute stroke unit care. Stroke 2004; 35:1005-1009.

9. Krakauer JW, Carmichael ST, Corbett D, Wittenberg GF. Getting neurorehabilitation right - what can we learn from animal models? Neurorehabil Neural Repair 2012; 26:923-931.

10. Hebb D. The Organization of Behavior. New York: John Wiley \& Sons Inc.; 1949.

11. Ohlsson AL, Johansson BB. Environment influences functional outcome of cerebral infarction in rats. Stroke 1995; 26:644-649.

12. Kolb B, Gibb R. Environmental enrichment and cortical injury: behavioral and anatomical consequences of frontal cortex lesions. Cereb Cortex 1991; 1:189-198.

13. Grabowski M, Brundin P, Johansson BB. Paw-reaching, sensorimotor, and rotational behavior after brain infarction in rats. Stroke 1993; 24:889-895.

14. Biernaskie J, Corbett D. Enriched rehabilitative training promotes improved forelimb motor function and enhanced dendritic growth after focal ischemic injury. J Neurosci 2001; 21:5272-5280.

15. Corbett D, Jeffers M, Nguemeni C, Gomez-Smith M, LivingstonThomas J. Lost in translation: rethinking approaches to stroke recovery. Prog Brain Res 2015; 218:413-434.

16. Jones TA, Schallert T. Use-dependent growth of pyramidal neurons after neocortical damage. J Neurosci 1994; 14:2140-2152.

17. Johansson BB, Belichenko PV. Neuronal plasticity and dendritic spines: effect of environmental enrichment on intact and postischemic rat brain. J Cereb Blood Flow Metab 2002; 22:89-96.

18. Clarke J, Langdon KD, Corbett D. Early poststroke experience differentially alters periinfarct layer II and III cortex. J Cereb Blood Flow Metab 2014; 34:630-637.

19. Dancause N, Nudo R. Shaping plasticity to enhance recovery after injury. Prog Brain Res 2011; 192:273-295.

20. Austin MW, Ploughman M, Glynn L, Corbett D. Aerobic exercise effects on neuroprotection and brain repair following stroke: a systematic review and perspective. Neurosci Res 2014; 87:8-15.

21. Garcia JH, Yoshida Y, Chen H, et al. Progression from ischemic injury to infarct following middle cerebral artery occlusion in the rat. Am J Pathol 1993; 142:623-635.

22. Maldonado MA, Allred RP, Felthauser EL, Jones TA. Motor skill training, but not voluntary exercise, improves skilled reaching after unilateral ischemic lesions of the sensorimotor cortex in rats. Neurorehabil Neural Repair 2008; 22:250-261.

23. Leasure JL, Grider M. The effect of mild post-stroke exercise on reactive neurogenesis and recovery of somatosensation in aged rats. Exp Neurol 2010; 226:58-67.

24. Shih PC, Yang YR, Wang RY. Effects of exercise intensity on spatial memory performance and hippocampal synaptic plasticity in transient brain ischemic rats. PLoS One 2013; 8:e78163.

25. Zhang QW, Deng XX, Sun X, Xu JX, Sun FY. Exercise promotes axon regeneration of newborn striatonigral and corticonigral projection neurons in rats after ischemic stroke. PLoS One 2013; 8 e80139.

26. Pang MY, Charlesworth SA, Lau RW, Chung RC. Using aerobic exercise to improve health outcomes and quality of life in stroke: evidence-based exercise prescription recommendations. Cerebrovasc Dis 2013; 35:7-22.

27. Billinger SA, Arena R, Bernhardt J, et al. Physical activity and exercise recommendations for stroke survivors: a statement for healthcare professionals from the American Heart Association/ American Stroke Association. Stroke 2014; 45:2532-2553.

28. Eng JJ. Fitness and Mobility Exercise (FAME Program for stroke). Top Geriatr Rehabil 2010; 26:310-323.

29. Marzolini S, Oh P, McIlroy W, Brooks D. The effects of an aerobic and resistance exercise training program on cognition following stroke. Neurorehabil Neural Repair 2013; 27:392-402.

30. Patel MD, Coshall C, Rudd AG, Wolfe C. Cognitive impairment after stroke: clinical determinants and its associations with longterm stroke outcomes. J Am Geriatr Soc 2002; 50:700-706.

31. Douiri A, Rudd AG, Wolfe C. Prevalence of poststroke cognitive impairment: South London Stroke Register 1995-2010. Stroke 2013; 44:138-145.

32. Pohjasvaara T, Leskelä M, Vataja R, et al. Post-stroke depression, executive dysfunction and functional outcome. Eur J Neurol 2002; 9:269-275.

33. Gobbo OL, O'Mara SM. Impact of enriched-environment housing on brain-derived neurotrophic factor and on cognitive performance after a transient global ischemia. Behav Brain Res 2004; 152:231241.

34. Farrell R, Evans S, Corbett D. Environmental enrichment enhances recovery but exacerbates ischemic cell death. Neuroscience 2001; 107:585-592.

35. Dahlqvist P, Ronnback A, Bergstrom SA, Söderström I, Olsson T. Environmental enrichment reverses learning impairment in the Morris water maze after focal cerebral ischemia in rats. Eur J Neurosci 2004; 19:2288-2298.

36. Wurm F, Keiner S, Kunze A, Witte OW, Redecker C. Effects of skilled forelimb training on hippocampal neurogenesis and spatial learning after focal cortical infarcts in the adult rat brain. Stroke 2007; 38:2833-2840.

37. Ekstrand J, Hellsten J, Tingström A. Environmental enrichment, exercise and corticosterone affect endothelial cell proliferation in adult rat hippocampus and prefrontal cortex. Neurosci Lett 2008; 442:203-207.

38. Rhyu I, Bytheway J, Kohler S, et al. Effects of aerobic exercise training on cognitive function and cortical vasularity in monkeys. Neuroscience 2010; 167:1239-1248.

39. Eng JJ, Reime B. Exercise for depressive symptoms in stroke patients: a systematic review and meta-analysis. Clin Rehabil 2014; 28: 731-739.

40. Jha S, Dong B, Sakata K. Enriched environment treatment reverses depression-like behavior and restores reduced hippocampal neurogenesis and protein levels of brain-derived neurotrophic factor in mice lacking its expression through promoter IV. Transl Psychiatry 2011; $1: e 40$.

41. Cramer SC. Repairing the human brain after stroke: I. Mechanisms of spontaneous recovery. Ann Neurol 2008, 63:272-287.

42. Cordova C, Jackson D, Langdon K, Hewlett K, Corbett D. Impaired executive function following ischemic stroke in the rat medial prefrontal cortex. Behav Brain Res 2014; 258:106-111.

43. Hewlett KA, Kelly MH, Corbett D. "Not-so-minor" stroke: Lasting psychosocial consequences of anterior cingulate cortical ischemia in the rat. Exp Neurol 2014; 261:543-550.

44. El-Tamawy MS, Abd-Allah F, Ahmed SM, Darwish MH, Khalifa HA. Aerobic exercises enhance cognitive functions and brain derived neurotrophic factor in ischemic stroke patients. Neurorehabilitation 2014; 34:209-213. 
45. Kim MW, Bang MS, Han TR, et al. Exercise increased BDNF and trkB in the contralateral hemisphere of the ischemic rat brain. Brain Res 2005; 1052:16-21.

46. Ploughman M, Attwood Z, White N, Doré JJ, Corbett D. Endurance exercise facilitates relearning of forelimb motor skill after focal ischemia. Eur J Neurosci 2007; 25:3453-3460.

47. Ploughman M, Windle V, MacLellan CL, White N, Doré JJ, Corbett D. Brain-derived neurotrophic factor contributes to recovery of skilled reaching after focal ischemia in rats. Stroke 2009; 40: 1490-1495.

48. Clarkson AN, Overman JJ, Zhong S, Mueller R, Lynch G, Carmichael ST. AMPA receptor-induced local brain-derived neurotrophic factor signaling mediates motor recovery after stroke. $\mathrm{J}$ Neurosci 2011; 31:3766-3775.

49. Mizutani K, Sonoda S, Yamada K, Beppu H, Shimpo K. Alteration of protein expression profile following voluntary exercise in the perilesional cortex of rats with focal cerebral infarction. Brain Res 2011; 1416:61-68.

50. Liu N, Huang H, Lin F, et al. Effects of treadmill exercise on the expression of netrin-1 and its receptors in rat brain after cerebral ischemia. Neuroscience 2011; 194:349-358.

51. Quirié A, Hervieu M, Garnier P, et al. Comparative effect of treadmill exercise on mature BDNF production in control versus stroke rats. PLoS One 2012; $7:$ e44218.

52. Hirata K, Kuge Y, Yokota C, et al. Gene and protein analysis of brain derived neurotrophic factor expression in relation to neurological recovery induced by an enriched environment in a rat stroke model. Neurosci Lett 2011; 495:210-215.

53. Jones TA, Chu CJ, Grande LA, Gregory AD. Motor skills training enhances lesion-induced structural plasticity in the motor cortex of adult rats. J Neurosci 1999; 19:10153-10163.

54. Gotts JE, Chesselet MF. Migration and fate of newly born cells after focal cortical ischemia in adult rats. J Neurosci Res 2005; 80:160 171.

55. Seo TB, Kim TW, Shin MS, et al. Aerobic exercise alleviates ischemia-induced memory impairment by enhancing cell proliferation and suppressing neuronal apoptosis in hippocampus. Int Neurourol J 2014; 18: 187-197.

56. Komitova M, Perfilieva E, Mattsson B, Eriksson PS, Johansson BB. Enriched environment after focal cortical ischemia enhances the generation of astroglia and NG2 positive polydendrocytes in adult rat neocortex. Exp Neurol 2006; 199:113-121.

57. Komitova M, Zhao LR, Gidö G, Johansson BB, Eriksson P. Postischemic exercise attenuates whereas enriched environment has certain enhancing effects on lesion-induced subventricular zone activation in the adult rat. Eur J Neurosci 2005; 21:2397-2405.

58. Bechara RG, Kelly ÁM. Exercise improves object recognition memory and induces BDNF expression and cell proliferation in cognitively enriched rats. Behav Brain Res 2013; 245:96-100.

59. Grégoire CA, Bonenfant D, Le Nguyen A, Aumont A, Fernandes $\mathrm{KJ}$. Untangling the influences of voluntary running, environmental complexity, social housing and stress on adult hippocampal neurogenesis. PLoS One 2014; 9: e86237.

60. Madinier A, Quattromani MJ, Sjölund C, Ruscher K, Wieloch T. Enriched housing enhances recovery of limb placement ability and reduces aggrecan-containing perineuronal nets in the rat somatosensory cortex after experimental stroke. PLoS One 2014; 9:e93121.

61. Li C, Wen H, Wang Q, et al. Exercise training inhibits the Nogo-A/ $\mathrm{NgR} 1 /$ Rho-A signals in the cortical peri-infarct area in hypertensive stroke rats. Am J Phys Med Rehabil 2015 94:1083-1094.

62. Papadopoulos CM, Tsai SY, Alsbiei T, O'Brien TE, Schwab ME, Kartje GL. Functional recovery and neuroanatomical plasticity following middle cerebral artery occlusion and $\mathrm{IN}-1$ antibody treatment in the adult rat. Ann Neurol 2002; 51:433-441.
63. Soleman S, Yip PK, Duricki DA, Moon LD. Delayed treatment with chondroitinase $\mathrm{ABC}$ promotes sensorimotor recovery and plasticity after stroke in aged rats. Brain 2012; 135:1210-1223.

64. Ma Y, Qiang L, He M. Exercise therapy augments the ischemiainduced proangiogenic state and results in sustained improvement after stroke. Int J Mol Sci 2013; 14:8570-8584.

65. Tian S, Zhang Y, Tian S, et al. Early exercise training improves ischemic outcome in rats by cerebral hemodynamics. Brain Res 2013; 1533:114-121.

66. Lang CE, Wagner JM, Edwards DF, Dromerick AW. Upper extremity use in people with hemiparesis in the first few weeks after stroke. J Neurol Phys Ther 2007; 31:56-63.

67. Taub E, Miller NE, Novack TA, et al. Technique to improve chronic motor deficit after stroke. Arch Phys Med Rehabil 1993; 74:347354.

68. Allred RP, Jones TA. Maladaptive effects of learning with the lessaffected forelimb after focal cortical infarcts in rats. Exp Neurol 2008; 210:172-181.

69. Maclellan CL, Langdon KD, Botsford A, Butt S, Corbett D. A model of persistent learned nonuse following focal ischemia in rats. Neurorehabil Neural Repair 2013; 27:900-907.

70. Allred RP, Cappellini CH, Jones TA. The "good" limb makes the "bad" limb worse: experience-dependent interhemispheric disruption of functional outcome after cortical infarcts in rats. Behav Neurosci 2010; 124:124-32.

71. Kim SY, Allred RP, Adkins DL, et al. Experience with the "good" limb induces aberrant synaptic plasticity in the perilesion cortex after stroke. J Neurosci 2015; 35:8604-8610.

72. Krueger H, Koot J, Hall RE, O'Callaghan C, Bayley M, Corbett D. Prevalence of individuals experiencing the effects of stroke in Canada: trends and projections. Stroke 2015; 46: 2226-2231.

73. Yager JY, Wright S, Armstrong EA, Jahraus CM, Saucier DM. The influence of aging on recovery following ischemic brain damage. Behav Brain Res 2006; 173:171-180.

74. Nakayama H, Jorgensen HS, Raaschou HO, Olsen TS. The influence of age on stroke outcome: the Copenhagen stroke study. Stroke 1994; 25:808-813.

75. Rosen CL, Dinapoli VA, Nagamine T, Crocco T. Influence of age on stroke outcome following transient focal ischemia. J Neurosurg 2005; 103:687-694.

76. Chou IC, Trakht T, Signori C, et al. Behavioral/environmental intervention improves learning after cerebral hypoxia-ischemia in rats. Stroke 2001; 32:2192-2197.

77. Rojas JJ, Deniz BF, Miguel PM, et al. Effects of daily environmental enrichment on behavior and dendritic spine density in hippocampus following neonatal hypoxia-ischemia in the rat. Exp Neurol 2013; 241:25-33.

78. Pereira LO, Arteni NS, Petersen RC, da Rocha AP, Achaval M, Netto CA. Effects of daily environmental enrichment on memory deficits and brain injury following neonatal hypoxia-ischemia in the rat. Neurobiol Learn Mem 2007; 87:101-108.

79. Buchhold B, Mogoanta L, Suofu Y, et al. Environmental enrichment improves functional and neuropathological indices following stroke in young and aged rats. Restor Neurol Neurosci 2007; 25: 467-484.

80. Bennett JC, McRae PA, Levy LJ, Frick KM. Long-term continuous, but not daily, environmental enrichment reduces spatial memory decline in aged male mice. Neurobiol Learn Mem 2006; 85:139152 .

81. Janssen H, Ada L, Bernhardt J, et al. An enriched environment increases activity in stroke patients undergoing rehabilitation in a mixed rehabilitation unit: a pilot non-randomized controlled trial. Disabil Rehabil 2014; 36:255-262.

82. Brown AW, Marlowe KJ, Bjelke B. Age effect on motor recovery in a post-acute animal stroke model. Neurobiol Aging 2003; 24:607614. 
83. Alaverdashvili M, Whishaw IQ. Compensation aids skilled reaching in aging and in recovery from forelimb motor cortex stroke in the rat. Neuroscience 2010; 167:21-30.

84. Fisher M, Feuerstein G, Howells DW, et al. Update of the stroke therapy academic industry roundtable preclinical recommendations. Stroke 2009; 40:2244-2250.

85. Rodriguez-Colon SM, Mo J, Duan Y, et al. Metabolic syndrome clusters and the risk of incident stroke: the atherosclerosis risk in communities (ARIC) study. Stroke 2009; 40: 200-205.
86. Reeson P, Tennant KA, Gerrow K, et al. Delayed Inhibition of VEGF signaling after stroke attenuates blood-brain barrier breakdown and improves functional recovery in a comorbiditydependent manner. J Neurosci 2015; 35:5128-5143.

87. Birkenmeier RL, Prager EM, Lang CE. Translating animal doses of task-specific training to people with chronic stroke in 1-hour therapy sessions: a proof-of-concept study. Neurorehabil Neural Repair 2010; 24:620-635.

88. Iadecola C, Anrather J. Stroke research at a crossroad: asking the brain for directions. Nat Neurosci 2011; 14:1363-1368. 